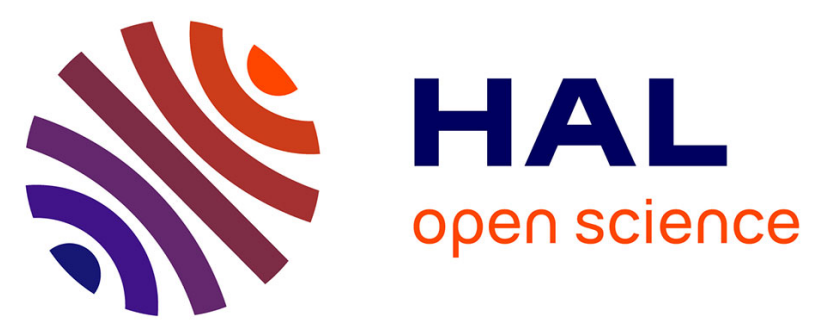

\title{
How exfoliated graphene oxide nanosheets organize at the water interface: evidence for a spontaneous bilayer self-assembly
}

Nathalie Bonatout, François Muller, Philippe Fontaine, Ignacio Gascon, Oleg Konovalov, Michel Goldmann

\section{To cite this version:}

Nathalie Bonatout, François Muller, Philippe Fontaine, Ignacio Gascon, Oleg Konovalov, et al.. How exfoliated graphene oxide nanosheets organize at the water interface: evidence for a spontaneous bilayer self-assembly. Nanoscale, 2017, 9 (34), pp.12543-12548. 10.1039/C7NR03403B . hal-01586796

\section{HAL Id: hal-01586796 \\ https://hal.sorbonne-universite.fr/hal-01586796}

Submitted on 13 Sep 2017

HAL is a multi-disciplinary open access archive for the deposit and dissemination of scientific research documents, whether they are published or not. The documents may come from teaching and research institutions in France or abroad, or from public or private research centers.
L'archive ouverte pluridisciplinaire HAL, est destinée au dépôt et à la diffusion de documents scientifiques de niveau recherche, publiés ou non, émanant des établissements d'enseignement et de recherche français ou étrangers, des laboratoires publics ou privés. 


\title{
Journal Name
}

\section{ARTICLE TYPE}

\section{How exfoliated Graphene Oxide nanosheets organize at water interface: evidences for a spontaneous bilayer self- assembly ${ }^{\dagger}$}

\author{
Nathalie Bonatout, ${ }^{a b}$ François Muller, ${ }^{b c}$ Philippe Fontaine, ${ }^{d}$ Ignacio Gascon, ${ }^{e}$ Oleg \\ Konovalov, ${ }^{f}$ and Michel Goldmann ${ }^{a d}$
}

\begin{abstract}
We probed Graphene Oxide adsorbed at the air-water interface by X-rays Reflectivity and Grazing Incidence X-Ray Diffraction using synchrotron sources. Surprisingly, the results of both measurements show that at non zero surface pressures, the film is organized as a bilayer of sheets interfaced between air and water with water molecule bridges. Such a spontaneous bilayer structure and its evolution with respect to the surface pressure is observed for the first time. These results should allow a precise control of the density of sheets deposited on the substrate when these films are transferred through the Langmuir-Blodgett or Schaefer procedures. Indeed, Graphene Oxide keeps on attracting more and more attention, increasing the need for the production of well-controlled Graphene Oxide thin films due to its interest for energy devices or in the sensor domains.
\end{abstract}

\section{Introduction}

Even though Graphene Oxide (GO) has been known for more than a century ${ }^{1,2}$, GO sheets have only been recently rising an increasing interest as they appear as a promising precursor to graphene based material. While graphene production processes are intensely studied ${ }^{3-5}$, the chemical difficulties and the high cost on the preparation of the pristine material still limit the use of graphene. These difficulties could be overcome through GO use and its reduction ${ }^{6,7}$. Furthermore, GO sheets have also been attracting more and more attention as they opens new horizons for advanced technological devices such as sensors ${ }^{8-10}$ or batteries ${ }^{11,12}$.

Good quality GO is commercially available and can be dispersed in polar solvents and then isolated as single layers ${ }^{13,14}$. The material can be further totally or partly reduced to increase its conductivity ${ }^{13}$. Local reduction using heated Atomic Force

\footnotetext{
a Sorbonne Universités, UPMC Univ Paris 06, CNRS-UMR 7588, Institut des NanoSciences de Paris, 75005 Paris, France. E-mail: michel.goldmann@insp.upmc.fr ${ }^{b}$ LICORNE, ECE Paris Ecole d'Ingénieurs, Immeuble POLLUX, 75015 Paris, France.

${ }^{c}$ LLB, CEA Saclay, 91191 Gif-sur-Yvette, France.

d Synchrotron SOLEIL L'Orme des Merisiers, Saint Aubin, 91192 Gif-sur-Yvette, France

e Physical Chemistry Department and Instituto de Nanociencia de Aragón, Universidad de Zaragoza, 50018 Zaragoza, Spain.

${ }^{f}$ ESRF-The European Synchrotron, 71, Avenue des Martyrs. 38100 Grenoble, France. $\dagger$ Electronic Supplementary Information (ESI) available. See DOI: 10.1039/b000000x/
}

Microscopy (AFM) tip has been successfully realized ${ }^{15}$, as well as direct laser reduction ${ }^{16}$. Many different configurations were proposed for the structure of GO colloidal plates. The chemical oxidation of graphite planes creates carboxylic acid, epoxide and hydroxyl groups on the produced exfoliated colloidal domains. The localization of these groups is a key parameter influencing the further organization of the material, since this drives the interactions between the GO plates. The most commonly assumed structure has been proposed by Lerf et al. ${ }^{17}$, placing the carboxylic acid groups on the edge of the sheets, the epoxide groups mainly in the middle of the sheets, and the hydroxyl groups both on the edge and in the middle of the sheets.

Intensive studies were carried out about transferring techniques for these GO sheets such as layer by layer deposition ${ }^{18,19}$, Langmuir Schaefer and Langmuir Blodgett (LB) techniques ${ }^{20-24}$, using the GO sheets as surfactants. The latter was used to deposit GO sheets monolayer on solid substrates at low surface pressures. Different studies were carried out on the specific organization of GO sheets deposited on solid substrates using X-Ray Diffraction ${ }^{25,26}$. The dynamics of water molecules in GO sheets have been investigated using neutron scattering 27,28 and the hydration of GO sheets has also been observed using Scanning Force Microscopy on transferred films ${ }^{29,30}$. These studies evidenced that water molecules get intercalated in GO thin films, increasing significantly the spacing between sheets. However, questions remain about the orientation, and the specific 
organization of the GO sheets at the air-water interface.

In this scope, we used a combination of Synchrotron techniques such as Reflectivity (XRR) and Grazing Incidence Diffraction (GIXD) to derive an in-situ structural model of GO sheets at the air-water interface. These experiments show that exfoliated GO sheets spontaneously form a bilayer with a buried layer lying flat at the water surface and a tilted top layer in contact with air. These structural properties are compared to the compressibility of the GO films .

\section{Methods}

Sample Preparation The GO aqueous dispersion was obtained from Sigma Aldrich and used without any further purification. The pristine dispersion concentration is of about $4 \mathrm{mg} \cdot \mathrm{mL}^{-1}$ and is characterized by a dark brown color. The GO sheets contain around $36 \%$ of oxygen, and the chemical formula is of the type $\mathrm{C}_{x} \mathrm{O}_{y} \mathrm{H}_{z}$.

In order to ensure the exfoliation of the anisotropic colloids, the dispersion was probe-sonicated for ten minutes and diluted in methanol (1:4) to allow its spreading on the surface water. Chloroform, which is typically used as solvent for Langmuir film deposition was not used to disperse GO since studies have shown that it tends to form aggregates of GO sheets when deposited at the water surface ${ }^{14,20}$. Before being slowly spread onto Milli-Q Millipore ultrapure water $(18.2 \mathrm{M} \Omega . \mathrm{cm})$ surface using a glass syringe, the solution was put in an ultrasonic bath for 15 minutes to further exfoliate graphite oxide to GO sheets. The isotherms obtained for $\mathrm{GO}$ were performed at $20^{\circ} \mathrm{C}$. The surface pressure was recorded using a Wilhelmy balance and Kirstein \& Riegler electronics at different compression speeds from $5 \mathrm{~cm} \cdot \mathrm{min}^{-1}$ up to $20 \mathrm{~cm}^{2} \cdot \mathrm{min}^{-1}$ without observing major change.

X-Ray Reflectivity experiments X-Ray Reflectivity experiments were carried out at the air-water interface on the ID10 beamline of the European Synchrotron Radiation Facility (ESRF France) using a Vantec detector. The energy of the incoming X-Ray beam was $22 \mathrm{keV}(\lambda=0.5636 \AA)$. The measurements were performed maintaining the surface pressure constant. The spectra were fitted according the Parratt formalism ${ }^{31}$ combined with genetic algorithm (Differential Evolution ${ }^{32}$ ) as the fitting routine, using the GenX $^{33}$ software. The Parratt formalism is a recursive algorithm used to model Fresnel reflectivity of a system of $N$ layers by expressing the reflectivity amplitude as a function of the scattering factor $r(q)$. Each layer is defined by its thickness $\Delta j$, roughness $\sigma_{j}$, electron density $\rho_{e, j}$ and scattering factor $f_{j}$.

Grazing Incidence X-Ray Diffraction experiments Grazing Incidence X-Ray Diffraction experiments were performed at the air-water interface on the SIRIUS beamline of the SOLEIL synchrotron source (France) with the usual setup described previously $^{34}$. The energy of the incoming X-Ray beam was $8 \mathrm{keV}$ $(\lambda=1.55 \AA)$. The scattering intensity was recorded using a vertical PSD associated to a Sollers slit collimator with a resolution of $0.002 \AA^{-1}$.

AFM experiments For the Atomic Force Microscopy measurements, LB GO films were transferred on vertical silicon wafers at a speed of $1 \mathrm{~mm} \cdot \mathrm{min}^{-1}$ and at a constant surface pressure. The substrates were washed in a Piranha bath (Sulfuric Acid/Hydrogen Peroxide 2:1) for 15 minutes. The measurements were performed using an AFM Dimension 3100 using a silicon cantilever in Tapping Mode with a spring constant of $40 \mathrm{~N} . \mathrm{m}^{-1}$.

\section{Results and discussion}

\subsection{Thermodynamical behavior}

A part of GO sheets sink into the subphase when they are spread at the air-water interface, due to their chemical structure (shown in Figure 1 (a)) and size variations. Kim et. al showed that decreasing the size of the GO platelets leads to increasing their hydrophilicity ${ }^{35}$. Therefore, the usual surface density parameter is undetermined, making the surface pressure the only reliable parameter for this system. This surface pressure is plotted here with respect to the trough area per milliliter of GO solution spread (once equilibrium is reached, GO films are nevertheless stable with respect to time as shown in the Supporting Information).

Figure 1 (b) shows a typical isotherm compression (Surface pressure $\pi$ versus trough area per milliliter of GO deposited $A$ ) of GO. Three different regions can be identified on the GO film isotherm, corresponding to different compressibilities. Indeed the compressibility of a film $C_{S}$ describes the packing of the film and the phases in presence ${ }^{36}$. It is defined as:

$$
C_{S}=-\frac{1}{A}\left(\frac{\delta A}{\delta \pi}\right)_{T}
$$

Figure 1 (c) displays the compressibility plotted against the surface pressure, (the surface pressure is the only reliable and relevant parameter for GO sheets). In the first region (i), the compressibility is diverging (thus not represented), indicating a two-phase coexistence plateau. At $\pi=0 \mathrm{mN} . \mathrm{m}^{-1}$, the layer can be considered as diluted in this region. After the lift-off of the surface pressure, up to $\pi=15 \mathrm{mN} \cdot \mathrm{m}^{-1}$, the compressibility decreases down to $C_{S}=33.4 m \cdot N^{-1}$ indicating a second region (ii). Then the film undergoes a change of compressibility which drops to $C_{S}=12.5 \mathrm{~m} . \mathrm{N}^{-1}$ at $\pi=40 \mathrm{mN} \cdot \mathrm{m}^{-1}$. This indicates a new condensed phase corresponding to a third region (iii). For a homogeneous system such as dipalmitoylphosphatidylcholine (DPPC), these compressibility values are in the range of the ones observed for the LE phase and for the LC phase at ambient temperature $^{37}$. However in the case of GO sheets, it may rather indicate the percolation of the GO sheets in region (ii) followed by the compression of a more homogeneous system in region (iii).

Using the LB procedures, GO films were transferred at different surface pressures on hydrophilic substrates. The transfer ratios were always characteristic of good transfer $(\tau>.9)$. AFM measurements are shown on Figure 1 (d-f). At $\pi=0 \mathrm{mN} \cdot \mathrm{m}^{-1}$ in region (i), the substrate is not entirely covered and single GO sheets can be observed. At $\pi=10 \mathrm{mN} \cdot \mathrm{m}^{-1}$ in region (ii), the coverage rate of the substrate increases and bilayers start to appear, at the edges of the GO sheets. Finally at $\pi=20 \mathrm{mN} . \mathrm{m}^{-1}$ in re- 

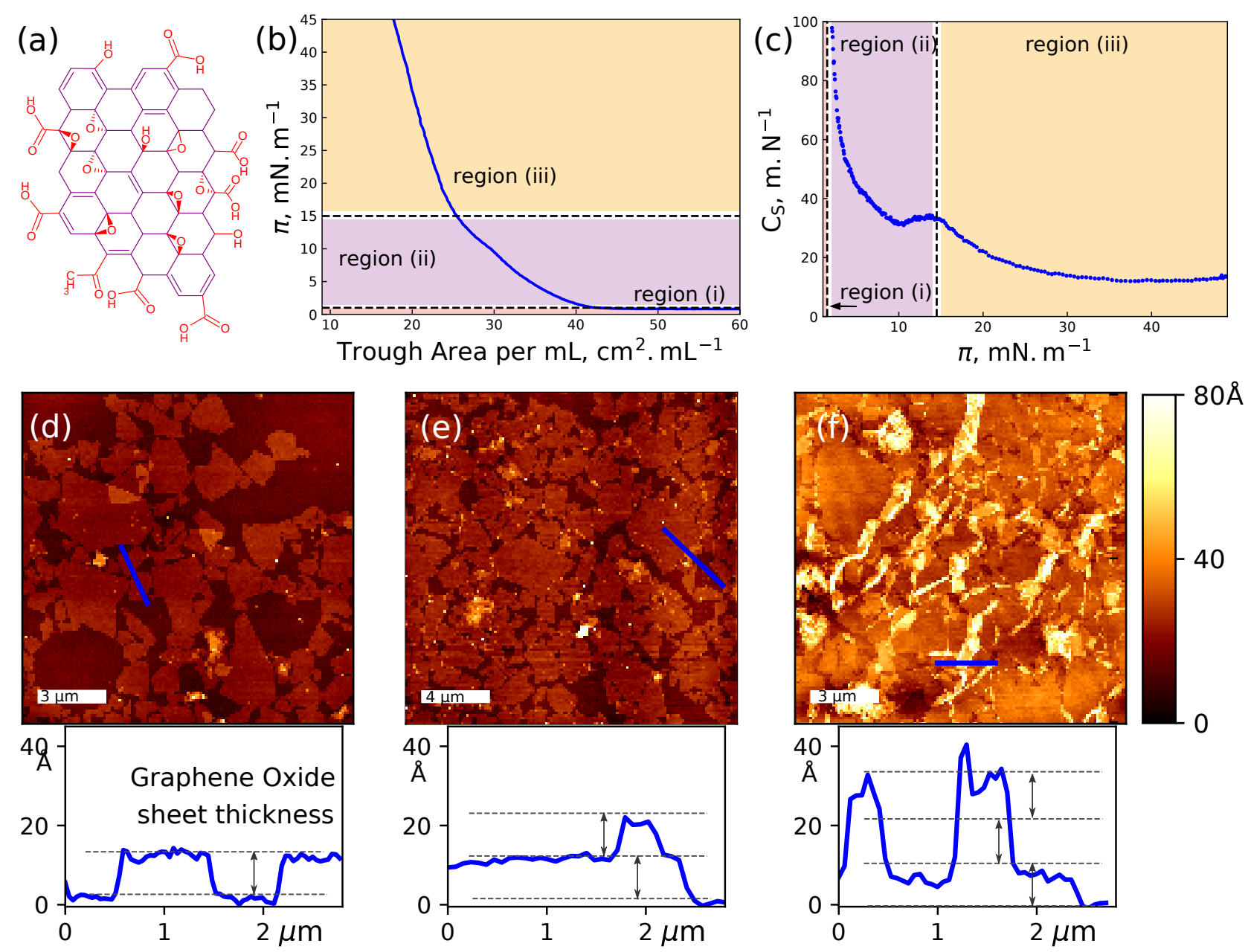

Fig. 1 (a) Chemical structure of GO sheets with hydrophobic groups in red and hydrophobic groups in purple adapted from Ref 14 . (b) $\pi-A$ isotherm of GO and (c) corresponding compressibility evolution with respect to $\pi$. AFM measurements on LB transferred GO films (d) at $\pi=0 \mathrm{mN} . \mathrm{m}^{-1}$, (e) at $\pi=10 \mathrm{mN} \cdot \mathrm{m}^{-1}$ and (f) at $\pi=20 \mathrm{mN} \cdot \mathrm{m}^{-1}$.

gion (iii), the substrate is entirely covered by GO sheets. Stacking starts to occur as bilayer and multilayer regions can be observed. The thickness of the bilayer is of about $20 \AA$, and the growth of small multilayer regions induces a high roughness.

\subsection{The bilayer formation}

Reflectivity experiments are sensitive to the variation of the electronic density perpendicular to the water surface. Reflectivity spectra recorded during the isotherm compression of GO films at fixed surface pressures are shown in Figure 2 (a) and (d). Working at the air-water interface, the hydration of GO sheets should be taken into account. It has been shown that GO sheets are permeable to water ${ }^{38}$ and that the thickness of the GO sheets tends to increase when they are in highly humid environments ${ }^{28,29}$. Different models were used to adjust the XRR spectra. We selected $\mathrm{C}_{2} \mathrm{O}_{8} \mathrm{H}_{.24}+1.4\left(\mathrm{H}_{2} \mathrm{O}\right)$ for the unit cell, where the presence of the water molecules is induced by the humid environment. This choice results from the experiments of Dreyer et al. ${ }^{14}$ and is based on the structure of GO sheet proposed by Lerf et al. ${ }^{17}$. It takes into account the presence of water molecules hydrogen bonded between the sheets as shown in Figure 2 (b-c).

Using this model, the fit leads to a layer about $20 \AA$ thick, a density of about $1.29 \mathrm{~g} . \mathrm{cm}^{-3}$ and a roughness of about $5 \AA$ at $\pi=0 \mathrm{mN} \cdot \mathrm{m}^{-1}$. It is known that the thickness of GO sheets is of about $10 \AA$ in humid environment ${ }^{28,29}$. Thus, we assumed that the overall surface layer of about $20 \AA$ resulting from the fit indicates a film composed of two layers of GO sheets. Such a bilayer was then used as a new model to adjust the reflectivity curves, by differentiating the different parameters for the two layers. The thicknesses of the model are accurate up to $\pm 1 \AA$.

To follow a possible system evolution with respect to time, XRR spectra were recorded over a period of five hours after the spreading of the GO sheets at the air-water interface. No major changes in the thickness, density and roughness were observed over this period (details can be found in the Supporting Information).

The evolution of the fitting parameters of the two layers model is shown in Figure $3(a-b)$.The two layers clearly differentiate 


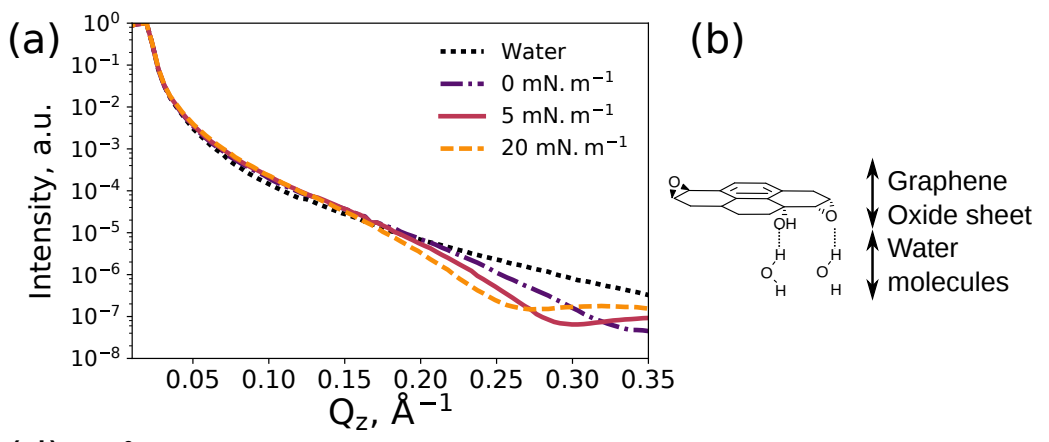

(c)

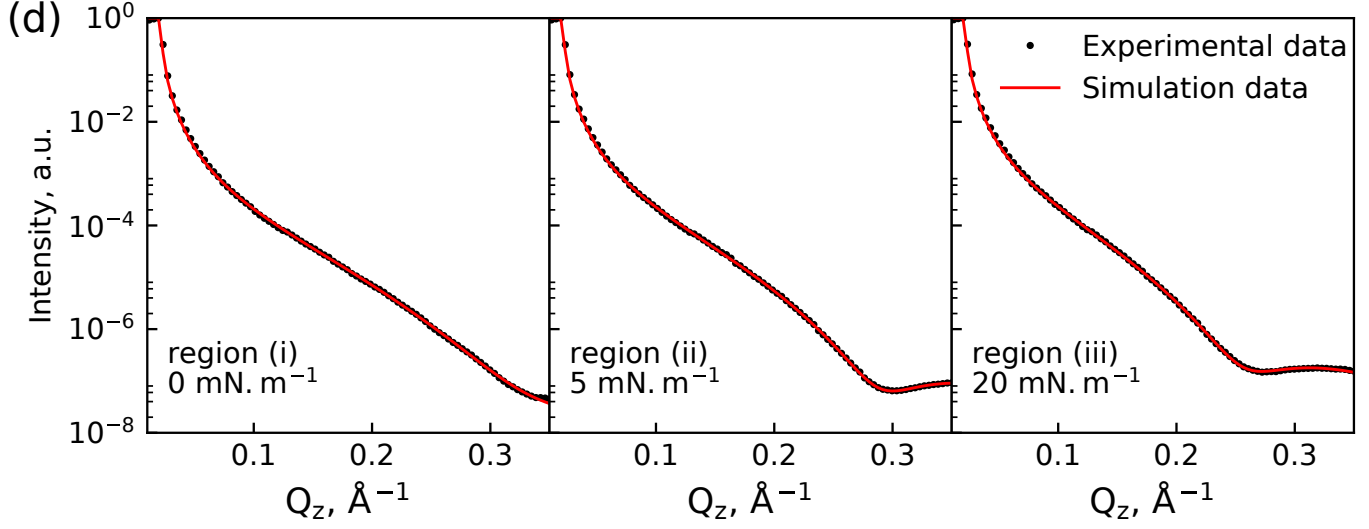

Fig. 2 (a) Reflectivity spectra of pure water and of GO sheets at the air-water interface at different $\pi$. Model of (b) the unit layer of GO sheet and (c) of the GO layers model at the air-water interface (adapted from Ref. 14). (d) Fitted XRR spectra in the three regions.

once the surface pressure starts rising. The thickness of the buried layer (in contact with the water subphase) does not change significantly and remains around $10 \AA$ when $\pi$ varies from 0 to $20 \mathrm{mN} . \mathrm{m}^{-1}$. Its roughness decreases from $5 \AA$ to $0.8 \AA$ and its density first increases from 1.3 up to $1.5 \mathrm{~g} . \mathrm{cm}^{-3}$ then remains constant. This clearly indicates a layer lying flat above the water surface, and getting denser when the surface pressure increases. The thickness of the top layer (in contact with air) increases from $10 \AA$ in region (i) to $12.5 \AA$ in region (iii). Its roughness increases up to $5 \AA$ and $6 \AA$, and its density of about $1.3 \mathrm{~g} \cdot \mathrm{cm}^{-3}$ in region (i), decreases down to around $1.1 \mathrm{~g} . \mathrm{cm}^{-3}$ in region (ii) and in region (iii). This top layer is then thicker and rougher that the buried one in region (iii) (above $\pi=15 \mathrm{mN} . \mathrm{m}^{-1}$ ) while its density is smaller.

\subsection{A tilted top layer}

GIXD spectra were recorded during the compression of the GO film adsorbed at the water surface at fixed surface pressures to probe the organization of the film in the plane parallel to the water surface.

No diffraction peak was observed before the surface pressure rises as shown in Figure 4 (a). This suggests either that the density of GO sheets at the water surface is too low to be detected at $\pi=0 \mathrm{mN} . \mathrm{m}^{-1}$ or that the layer is disorganized. However in region (ii) and region (iii) of the isotherm, two peaks were reproducibly observed. One of them , $(\alpha)$, is symmetric centered at $Q_{x y}=1.497 \AA^{-1}$ and at $Q_{z}=0 \AA^{-1}$, as shown in Figure 4 (b). The second peak, $(\beta)$, centered at $Q_{x y}=1.450 \AA^{-1}$ and (a)

(b)

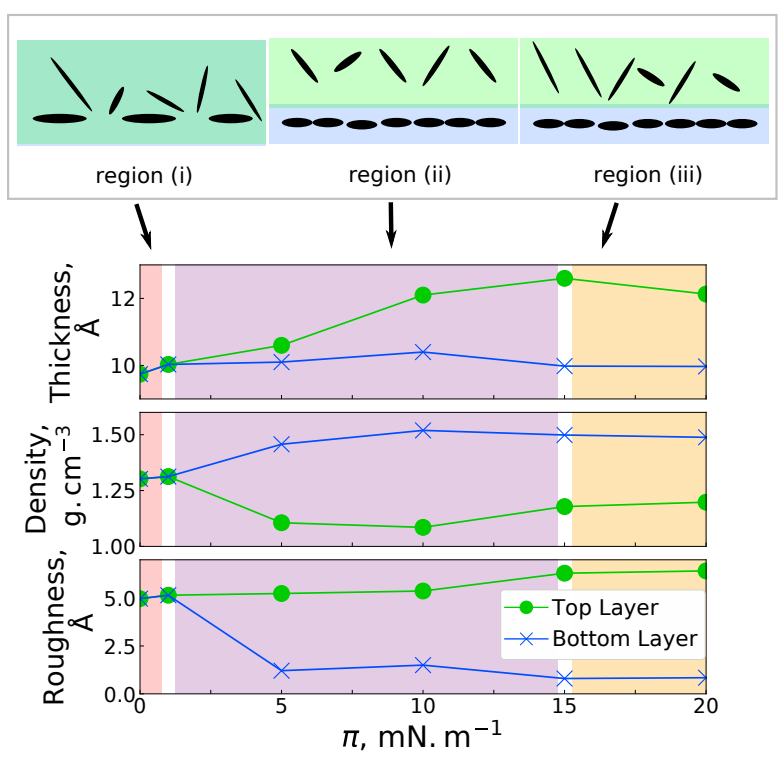

Fig. 3 (a) Schematic representation of the model proposed for the GO sheets at the air-water interface during the compression in region (i), region (ii) and region (iii). (b) Evolution of the thickness, the density and the roughness fitting parameters for the reflectivity spectra of GO sheets at the air-water interface with respect to $\pi$ using the bilayer model and the color code of Figure 2 (c).

at $Q_{z}=0.09 \AA^{-1}$ presents an asymmetrical shape with a tail extended towards higher $Q_{x y}$. Such a shape is typical of textured oriented lamellar structures, well-known as "Warren peak"39,40. Being a Warren peak, this peak $(\beta)$ suggests an in-plane organization of the GO film. As a consequence, the presence of such a 
diffraction peak located out-of-plane $\left(Q_{z}=0.09 \AA^{-1}\right)$ indicates that the diffracting structure has not its diffraction planes parallel to the water surface.

Theses results can be interpreted by the coexistence of two networks. One of them, corresponding to the symmetric peak $(\alpha)$, would lie parallel to the water surface with an average parameter $a_{\alpha}=4.20 \AA$ assuming a hexagonal network. The second one, corresponding to the asymmetric peak $(\beta)$, would be tilted with respect to the water surface but with a less-defined orientation, with an average parameter $a_{\beta}=4.31 \AA$ using the same assumption.

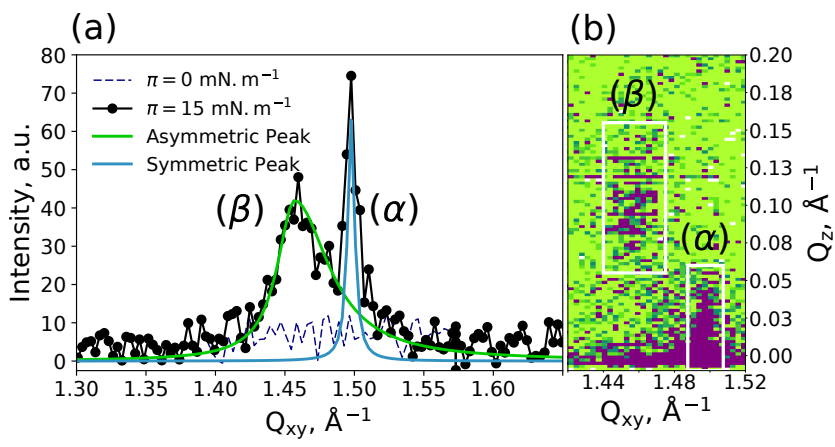

Fig. 4 (a) Diffraction pattern of GO with fitted peaks at the air-water interface at $\pi=15 \mathrm{mN} . \mathrm{m}^{-1}$ and at $\pi=0 \mathrm{mN} . \mathrm{m}^{-1}$. (b) Reciprocal space map of GO sheets at the air-water interface at $\pi=15 \mathrm{mN} . \mathrm{m}^{-1}$.

\subsection{The in-situ structural model}

Gathering the isotherm, the XRR and GIXD results, an overall model can be proposed for the GO sheets behavior along compression: at very low surface density, in region (i), the film is composed by dispersed GO sheets at the water surface either lying flat or with random orientations. When the surface density is high enough, up to $\pi=15 \mathrm{mN} . \mathrm{m}^{-1}$ in region (ii), the film is formed by a bilayer of GO sheets with a thickness of around $10 \AA$ each. The high compressibility $C_{S}=33.4 \mathrm{~m} \cdot \mathrm{N}^{-1}$ is coherent with the climbing process of the upper sheets. In region (iii), at higher surface pressures, the tilted layer gets thicker, denser and rougher while the buried layer is formed by flat sheets at the water surface.

These layers can be associated to the diffraction peaks observed at $Q_{x y}=1.497 \AA^{-1}$ and at $Q_{x y}=1.450 \AA^{-1}$. The first peak is associated to the buried GO layer and indicates a layer lying flat with respect to the water surface. The second peak shape indicates a texturation of the GO sheets associated to their disorientation with respect to the water surface. It can be associated with the top GO layer of the XRR model. The climb of GO sheets under compression leads to a less ordered upper layer. The low compressibility $C_{S}=12.5 \mathrm{~m} \cdot \mathrm{N}^{-1}$ corresponds to the region where the structure of the lower layer cannot be any more modified while the out-of-plane upper layer is still reorganized. Moreover, the lattice expansion of the upper layer associated peak is coherent with its density decrease deduced from our XRR model: upper GO sheets are less dense but they occupy a larger volume than buried GO sheets (details available in the Supporting Information).

Looking at the AFM measurements performed for LB transfers in the (i) and (ii) regions, only one layer of GO sheets is transferred, while XRR and GIXD and measurements are in agreement with a two layer model at the air-water interface. The films at the air-water interface are too dense to allow the splitting of the bilayer in a sparse single layer without any stacking of the GO sheets as can be observed for transfers at $\pi=0 \mathrm{mN} . \mathrm{m}^{-1}$. One possible explanation is the drainage mechanism when transferring the film: only the sheets parallel to the water surface are effectively transferred on the substrate. This suggests that the hydration plays a central role on the transfer process and thus cannot be neglected when working with GO sheets. The hydration of the sheets appears to be the effect that regulates the structure.

It is obvious that the interfacial energy is the main physical parameter driving the mechanism of organization at the interface. This has already been show at the oil-water interface through the observation of stabilized Pickering emulsion using organic solvents ${ }^{41}$. As surfactants, GO sheets have a particular chemical structure since the hydrophobic and the hydrophilic groups are nearly in the same plane due to their asymmetrical plate shape. This implies that lying flat at the interface is not favorable and has a large energy cost because both hydrophilic and hydrophobic domains are simultaneously in contact with water and air, which induces a frustration in the film. A bilayer organization using water molecules as lift as experimentally observed is one of the way to decrease this surface of contact. Furthermore the results we obtained show that this bilayer formation happens spontaneously at the air-water interface. This tends to prove that the GO sheets are effectively well exfoliated when deposited at the interface. Detailed theoretical calculations deserve to be developed in order to investigate further the mechanism of the bilayer formation and show why the bilayer is the optimal structure in terms of energy.

\section{Conclusions}

An in-situ structural model has been proposed to describe GO sheets behavior along compression at the air-water interface using a combination of Synchrotron techniques. Surprisingly, even at very low surface density (region (i)), the film is composed of a bilayer of dispersed GO sheets at the water surface. When the surface density is high enough (region (ii)), the film is formed by a bilayer of GO sheets, both of them having different roughnesses, thicknesses and densities. One layer is in contact with water and contains GO sheets lying parallel to the water surface. The second layer, in contact with air, is formed by GO sheets tilted with respect to the water surface. Finally in region (iii), the upper layer thickness, density and roughness increase, while the buried layer remains flat at the water surface. 


\section{Acknowledgement}

This work was financially supported by a DGA-MRIS scholarship (Contract No. 2014.60.0031) and ECE Paris. The authors thank the SIRIUS beamline of SOLEIL Synchrotron and the ID10 beamline of ESRF Synchrotron. The authors also thank L. Bondaz for his help on the experiments at the ESRF Synchrotron.

\section{References}

1 B. Brodie, 1859.

2 W. S. Hummers and R. E. Offeman, Journal of the American Chemical Society, 1958, 80, 1339-1339.

3 N. J. Walch, A. Nabok, F. Davis and S. P. J. Higson, Beilstein Journal of Nanotechnology, 2016, 7, 209-219.

4 M. Lotya, Y. Hernandez, P. J. King, R. J. Smith, V. Nicolosi, L. S. Karlsson, M. Blighe, S. De, Z. Wang, I. T. Mcgovern, G. S. Duesberg, J. N. Coleman and F. M. Blighe, 2009, 3611-3620.

5 A. T. Najafabadi and E. Gyenge, Carbon, 2014, 71, 58-69.

6 T. Kuila, A. K. Mishra, P. Khanra, N. H. Kim and J. H. Lee, Nanoscale, 2013, 5, 52-71.

7 S. Pei and H. M. Cheng, Carbon, 2012, 50, 3210-3228.

8 M. Pumera, Chemical record (New York, N.Y.), 2009, 9, 21123.

9 K. Toda, R. Furue and S. Hayami, Analytica Chimica Acta, 2015, 878, 43-53.

10 S. Borini, R. White, D. Wei, M. Astley, S. Haque, E. Spigone, N. Harris, J. Kivioja and T. Ryhä, 2013, 7, 11166-11173.

11 G. P. Keeley, A. O'Neill, N. McEvoy, N. Peltekis, J. N. Coleman and G. S. Duesberg, Journal of Materials Chemistry, 2010, 20, 7864-7869.

12 B. Xu, S. Yue, Z. Sui, X. Zhang, S. Hou, G. Cao and Y. Yang, Energy \& Environmental Science, 2011, 4, 2826.

13 D. Li, M. B. Muller, S. Gilje, R. B. Kaner and G. G. Wallace, Nature Nanotechnology, 2008, 3, 101-105.

14 D. R. Dreyer, S. Park, C. W. Bielawski and R. S. Ruoff, Chemical Society reviews, 2010, 39, 228-240.

15 Z. Wei, D. Wang, S. S.-Y. Kim, S. S.-Y. Kim, Y. Hu, M. K. Yakes, A. R. Laracuente, Z. Dai, S. R. Marder, C. Berger, W. P. King, W. A. de Heer, P. E. Sheehan, E. Riedo, W. A. de Heer, P. E. Sheehan and E. Riedo, Science, 2010, 328, 1373-1376.

16 R. Trusovas, K. Ratautas, G. Račiukaitis, J. Barkauskas, I. Stankevičiene, G. Niaura and R. Mažeikiene, Carbon, 2013, 52, 574-582.

17 H. Y. He, J. Klinowski, M. Forster and A. Lerf, Chemical Physics Letters, 1998, 287, 53-56.

18 J. Shen, Y. Hu, C. Li, C. Qin, M. Shi and M. Ye, Langmuir, 2009, 25, 6122-6128.

19 N. I. Kovtyukhova, P. J. Ollivier, B. R. Martin, T. E. Mallouk,
S. a. Chizhik, E. V. Buzaneva and A. D. Gorchinskiy, Chemistry of Materials, 1999, 11, 771-778.

20 L. J. Cote, F. Kim and J. Huang, Journal of the American Chemical Society, 2009, 131, 1043-1049.

21 R. S. Hidalgo, D. López-Díaz and M. M. Velázquez, Langmuir, 2015, 31, 2697-2705.

22 Q. Zheng, B. Zhang, X. Lin, X. Shen, N. Yousefi, Z.-D. Huang, Z. Li and J.-K. Kim, Journal of Materials Chemistry, 2012, 22, 25072.

23 X. Li, G. Zhang, X. Bai, X. Sun, X. Wang, E. Wang and H. Dai, Nature nanotechnology, 2008, 3, 538-542.

24 K. L. Harrison, L. B. Biedermann and K. R. Zavadil, Langmuir, 2015, 31, 9825-9832.

25 S.-J. Mu, Y.-C. Su, L.-H. Xiao, S.-D. Liu, T. Hu and H.-B. Tang, Chinese Physics Letters, 2013, 30, 096101.

26 D. a. Dikin, S. Stankovich, E. J. Zimney, R. D. Piner, G. H. B. Dommett, G. Evmenenko, S. T. Nguyen and R. S. Ruoff, Nature, 2007, 448, 457-460.

27 A. Buchsteiner, A. Lerf and J. Pieper, Journal of Physical Chemistry $B, 2006,110,22328-22338$.

28 A. Vorobiev, A. Dennison, D. Chernyshov, V. Skrypnychuk, D. Barbero and A. V. Talyzin, Nanoscale, 2014, 6, 1215112156.

29 B. Rezania, N. Severin, A. V. Talyzin and J. P. Rabe, Nano Letters, 2014, 14, 3993-3998.

30 J. I. Paredes, S. Villar-Rodil, P. Solís-Fernández, a. MartínezAlonso and J. M. D. Tascón, Langmuir, 2009, 25, 5957-5968.

31 L. G. Parratt, Physical Review, 1954, 95, 359-369.

32 M. Björck, Journal of Applied Crystallography, 2011, 44 1198-1204.

33 M. Björck and G. Andersson, Journal of Applied Crystallography, 2007, 40, 1174-1178.

34 P. Fontaine, M. Goldmann, M. Bordessoule and A. Jucha, Review of Scientific Instruments, 2004, 75, 3097-3106.

35 J. Kim, L. J. Cote, F. Kim, W. Yuan, K. Shull and J. J. Huang, Journal of the American Chemical Society, 2010, 132, 81808186.

36 M. C. Petty, Langmuir-Blodgett films, Cambridge University Press, Cambridge, 1996.

37 S. L. Duncan and R. G. Larson, Biophysical journal, 2008, 94, 2965-2986.

38 R. R. Nair, H. A. Wu, P. N. Jayaram, V. Grigorieva and A. K. Geim, Science, 2012, 335, 442-444.

39 B. E. Warren, Physical Review, 1941, 59, 693-698.

40 K. a. Carrado, Clay Minerals, 1997, 32, 29-40.

41 F. Kim, L. J. Cote and J. Huang, Advanced Materials, 2010, 22 , 1954-1958. 